\title{
Integrating ecological theories and traits in process-based modeling of macroinvertebrate community dynamics in streams
}

\author{
Cédric P. Mondy ${ }^{1}$ And Nele Schuwirth \\ Eawag, Swiss Federal Institute of Aquatic Science and Technology, 8600, Dübendorf, Switzerland
}

\begin{abstract}
Predicting the composition and dynamics of communities is a challenging but useful task to efficiently support ecosystem management. Community ecology has developed a number of promising theories, including food webs, metabolic theory, ecological stoichiometry, and environmental filtering. Their joint implementation in a mechanistic modeling framework should help us to bring community ecology to a new level by improving its predictive abilities. One of the challenges lies in the proper consideration of model uncertainty. In this paper, we contribute to this challenging task by modeling the temporal dynamics of macroinvertebrate communities in a stream subjected to hydropeaking in Switzerland. To this end, we extended the mechanistic model Streambugs regarding flood-induced drift processes and the use of trait information to define performance filters. Model predictions without any calibration were in the right order of magnitude but did not reflect the dynamics of most of the invertebrate taxa well. Bayesian inference drastically improved the model fit. It revealed that a large share of total model output uncertainty can be attributed to observation errors, which exceeded model parameter uncertainty. Observed and simulated community-aggregated traits helped to identify and understand model deficits. The combination of different ecological theories and trait information in a single mechanistic modeling framework combined with Bayesian inference can thus help to predict responses of communities to environmental changes, which can support ecosystem management.
\end{abstract}

Key words: Bayesian inference; community assembly; food web; macroinvertebrates; mechanistic model; traits; uncertainty analysis.

\section{INTRODUCTION}

Community ecology looks for laws or general hypotheses that explain the patterns describing the assemblages of co-occurring species among one or several trophic levels (MacArthur 1984), an achievement of the utmost importance to predict the consequences of various forms of pollution and to support different management options dealing with those problems. The existence of such laws has been heavily debated (Lawton 1999, Scheiner and Willig 2008). McGill et al. (2006) argued that this doubt about the existence of laws in community ecology results from the overwhelming focus of ecologists on the taxonomic description of communities and on biotic interactions as opposed to the description of organisms' functional characteristics, or traits, and the relationships with their environment. Shifting this focus could help improve our understanding of the mechanisms driving communities (McGill et al. 2006) and food webs (Ings et al. 2009). Despite this conceptual background, community ecology still lacks predictive power (Ings et al. 2009) and our comprehension of community response to environmental changes is limited (Brown et al. 2011). A potential explanation is the over-representation of pattern

Manuscript received 14 March 2016; revised 23 January 2017; accepted 8 February 2017. Corresponding Editor: John C. Stella.

${ }^{1}$ E-mail: cedric.mondy@gmail.com descriptions in the literature compared to the investigation of the underlying processes (Schoener 1986, Emmerson 2011).

Process-based models translate mechanistic hypotheses into mathematical expressions of the processes and produce predictions that can be used to test the validity and generality of these hypotheses (Kendall et al. 1999). During the last decade several attempts to improve the predictive abilities of community ecology have been made using principles from stoichiometry (Moe et al. 2005), allometry (Petchey et al. 2008), the metabolic theory of ecology (MTE; Brown et al. 2004, Price et al. 2010) or traits (Shipley et al. 2006, Webb et al. 2010). Despite the progress made, the models have so far been limited to some particular aspects of the communities, e.g., community-aggregated trait profiles (Shipley et al. 2006, Webb et al. 2010), individual populations abundances (Ott et al. 2014), functional groups (Schuwirth et al. 2008), or food web links (Petchey et al. 2008). The quantitative prediction of the populations making up communities and food webs is still rare (Ings et al. 2009).

The key to taking the next step toward predictive community ecology may be the integration of these different theories/principles in one single framework: (1) food web ecology explaining the relationships between consumers and their food sources and the trophic organization of communities (Pimm 1982), (2) ecological stoichiometry considering how the elemental composition of consumers 
and food sources influence the energy and organic matter fluxes and therefore the biological rates of consumers (Sterner and Elser 2002), (3) MTE and allometric scaling linking individual organism characteristics (e.g., body size) to biological processes (Brown et al. 2004), and (4) traits describing how environmental conditions act as filters to select fitting organisms (McGill et al. 2006). In recent years, promising modeling approaches have emerged. Community ecologists have combined food web principles and allometric relationships (Petchey et al. 2008, Boit et al. 2012) or MTE and stoichiometry (Allen and Gillooly 2009, Ott et al. 2014) to predict community features. In trait-based ecology, the concepts of "performance filters" (Webb et al. 2010) and intraspecific trait variation have been incorporated into a framework to predict the relative abundance of nine tree species (Laughlin et al. 2012). To our knowledge, the Streambugs model (Schuwirth and Reichert 2013) is the first attempt to combine MTE/stoichiometry (that focus on organism properties) and trait-based approaches (that focus on the role of the environment as a filter for organisms) in one model framework.

Streambugs is a process-based food-web model that is used to predict the macroinvertebrate community composition in streams by (1) estimating the occurrence probability of taxa under constant environmental conditions (Schuwirth and Reichert 2013, Schuwirth et al. 2016) and (2) estimating community dynamics in mesocosms with pesticide contamination (Kattwinkel et al. 2016). The model is formulated as a set of ordinary differential equations that describe taxon biomass dynamics dependent on population processes (growth, death, respiration). These processes are formulated as a function of environmental influence factors and taxon specific traits.

Mechanistic ecological models such as Streambugs require the specification of numerous parameters, for many of which prior uncertainty is quite large. Inferring these parameters from field observations of the state variables (i.e., model calibration) and assessing model output uncertainty is therefore an important challenge, especially if it is intended to support ecosystem management (Ascough et al. 2008). Bayesian methods offer a consistent framework to jointly address both issues and, applied to mechanistic models, hold great promise for advancing predictive ecology (Webb et al. 2010).

Our aims in this study were to (1) extend the Streambugs model regarding short term disturbances (floods) to model the dynamic development of taxa abundances over time in natural systems, (2) generalize the integration of trait information in the model adapting the performance filter concept, (3) apply the model to a stream with hydropeaking experiments to quantitatively predict macroinvertebrate community dynamics, (4) use Bayesian inference to jointly calibrate the model parameters and assess the prediction uncertainties, and (5) investigate how biological trait information can help to critically discuss the model results and to identify potential for future improvements.
Material And Methods

\section{Study site}

We used data on the temporal dynamics of invertebrates from the River Sihl, a Swiss river of about $68 \mathrm{~km}$ length, flowing from Drusberg in the prealps (Canton Schwyz) to the River Limmat in the city of Zurich. The data was collected at a site in the Swiss Plateau near Sihlwald (coordinates $684825 / 235$ 550, Swiss Grid) on behalf of the Canton of Zurich in the scope of hydropeaking experiments (Elber et al. unpublished data, Schuwirth et al. 2008). The river is subjected to flow abstraction for hydropower generation at the Sihlsee, which leads to an excessive biomass development of filamentous algae. The hydropeaking experiments were conducted to assess the extent of artificial floods needed to reduce algal biomass. At this site, the River Sihl is a betasaprobic stream with a width of about $20 \mathrm{~m}$, a slope of $0.67 \%$ (estimate with GIS), with about $10 \%$ of the stream bed shaded by surrounding trees (estimate from aerial pictures), and there is no known pesticide contamination. The derivation of environmental conditions is described in document $\mathrm{S} 1$ and the dynamics of the environmental conditions are given in Fig. 1.

A detailed description of the field site and sampling methods is given in Schuwirth et al. (2008). In short, the benthic communities (algae and invertebrates) of the river were investigated 26 times between April 1991 and July 1992. Algae were aggregated into the functional groups of filamentous (considered to be macrophytes) and crusty (considered to be microphytes) algae. Their ash free dry mass (AFDM) was estimated from total biomass (measured in $\mathrm{g}_{\mathrm{AFDM}} / \mathrm{m}^{2}$ ) and group-specific coverage data (Fig. 1). The filamentous algae mainly consisted of the green alga Cladophora glomerata (L.) Kützing and, to a lesser degree, of the green alga Ulothrix zonata (Weber et Mohr) Kützing. The functional group of crusty algae mainly consisted of the green alga Gongrosira incrustans (Reinsch) Schmidle, the bluegreen alga Homoeothrix varians Geitler, and several diatoms.

Invertebrates were sampled with a Surber net $(30 \times 30 \mathrm{~cm}$; mesh size of $280 \mu \mathrm{m})$ at six points along a transversal section of the river at the study site. The six samples were equally distributed across the width of the river. The samplings were performed about every second week (Table S2). To avoid effects from previous samplings, the transect was shifted $3 \mathrm{~m}$ upstream for each sampling. After every seventh sampling, the transect was shifted to its original location. Samples were pooled and invertebrates were then sorted, identified, and counted in the laboratory (Table S2). Taxa were identified to different taxonomic levels (from species to class). Taxonomic harmonization was applied to avoid identification bias: Dipterans and the Coleoptera family Elmidae were identified to family level; all other insect groups to genus level. Other organisms were discarded if not identified to family level, except Oligochaeta. Finally, we did not take into account rare taxa (Caenis, Dinocras, Psychodidae, and 

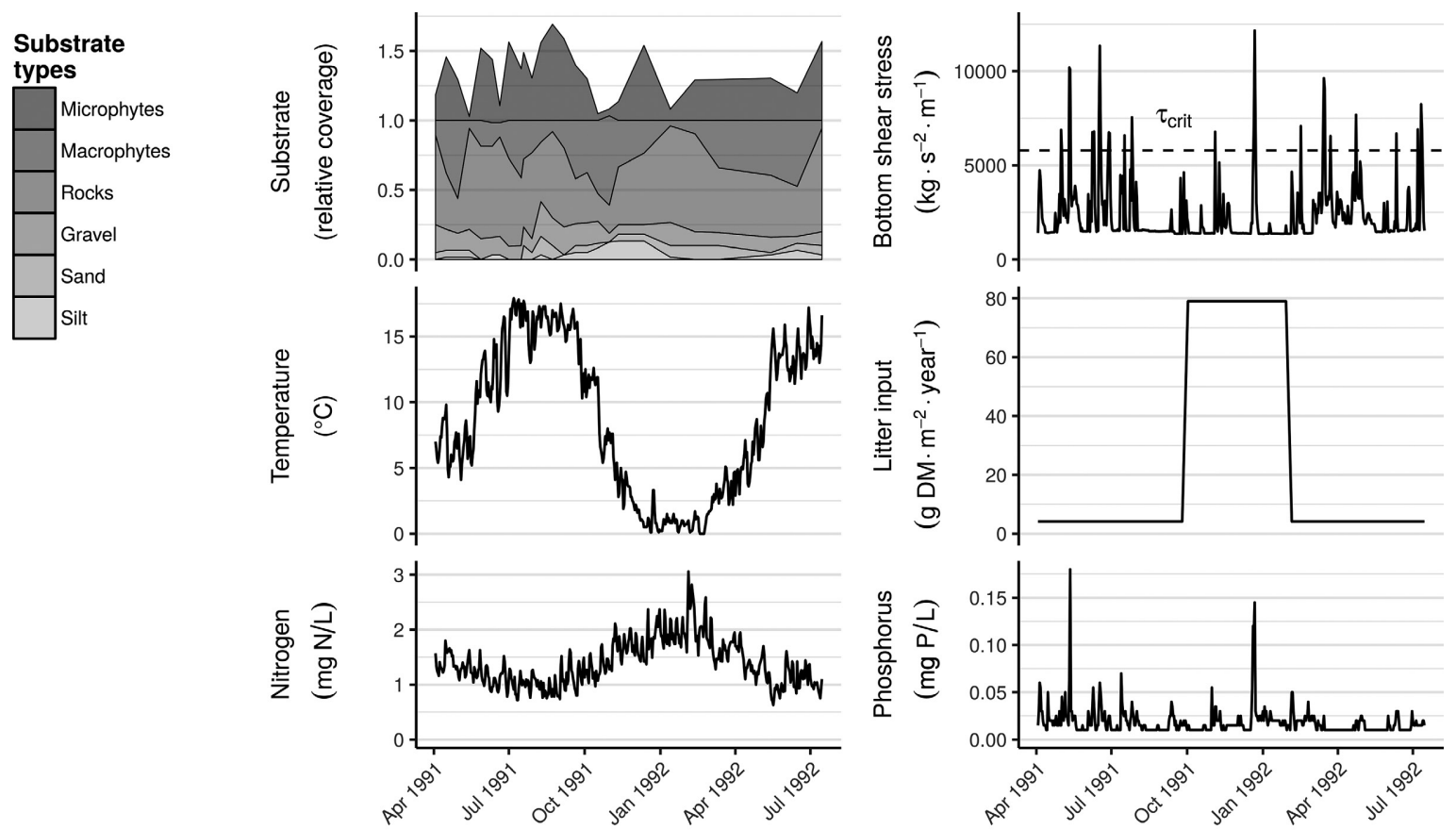

FIG. 1. Temporal evolution of environmental conditions in the Sihl. The dashed line shows $\tau_{\text {crit }}$, the calibrated value of the critical bottom shear stress above which flood-induced drift occurs. DM, dry mass.

Rhithrogena) with low occurrences (less than $25 \%$ of the 26 samples) and abundances (less than 50 individuals per sampled area on average) because their dynamics may be largely affected by demographic stochasticity, which we could not account for with our model approach (see Discussion). A total of 13 taxa were thus considered in the following analyses (Table S2).

\section{Streambugs implementation}

The full model description is given in the document S3. The model outputs are expressed in mass ( $\mathrm{g}$ of dry matter $\left.\left[g_{D M}\right]\right)$ per unit river length since this dimension is stable under changing water levels in contrast to mass per unit river surface.

Flood-induced drift process.-To take into account the effects of floods occurring in the River Sihl as a consequence of hydropeaking experiments, we implemented a drift process. The rate for this process (Eq. 1) is based on the formulation given in Schuwirth et al. (2008). We assumed (1) that biomass loss occurs above a certain bottom shear stress threshold $\tau_{\text {crit }}$ (see also Gibbins et al. 2007 ) and (2) that the rate of biomass loss $r_{\text {drift }}$ is proportional to the population biomass $B$, to the square of the difference between observed bottom shear stress $\tau$ and the $\tau_{\text {crit }}$ threshold, and to a taxon specific parameter $f_{\text {drift }}$

$$
r_{\text {drift }}= \begin{cases}0 & \text { if } \tau<\tau_{\text {crit }} \\ f_{\text {drift }} \times B \times\left(\tau-\tau_{\text {crit }}\right)^{2} & \text { if } \tau \geq \tau_{\text {crit }}\end{cases}
$$

Traits. - The average individual body mass, obtained from measures of individuals collected at the Swiss Plateau (Table S4), was used to calculate the basal metabolic rate according to Brown et al. (2004) (Eq. 2)

$$
\tilde{r}_{\text {basal }}=i_{0} \times\left(\frac{M}{M_{0}}\right)^{b} \times e^{\frac{-E_{A}}{k_{B} \times T}}
$$

The individual basal metabolic rate of organisms (in W or $\mathrm{J} / \mathrm{s}$ ) corresponds to the energy turnover per time and is calculated using a normalization constant $\left(i_{0}\right.$, in $\left.\mathrm{W}\right)$, the activation energy $E_{\mathrm{A}}$ (in electronvolts; $1 \mathrm{eV}=1.602 \times$ $10^{-19} \mathrm{~J}$ ), the body temperature $T$ (in $\mathrm{K}$ ), the Boltzmann's constant $k_{\mathrm{B}}\left(8.617 \times 10^{-5} \mathrm{eV} / \mathrm{K}\right)$, the individual body mass $M\left(\mathrm{~g}_{\mathrm{DM}}\right)$ and a standard body mass $\left(M_{0}=1 \mathrm{~g}_{\mathrm{DM}}\right)$. Since macroinvertebrates are small poikilothermic organisms, we assumed that they are in thermal equilibrium with the surrounding water and that the body temperature could thus be estimated by the water temperature.

In the following steps, we used fuzzy-coded ecological traits (Chevenet et al. 1994, Tachet et al. 2010) to modify growth and death processes to account for taxon specific differences. These traits describe different aspects of environmental requirements of taxa using fuzzy scores (from 0 , no affinity, to 3 or 5 , high affinity, depending on the underlying ecological complexity, and on the available amount of information) allocated to different categories corresponding to nominal categories (e.g., sand or cobble substrates) or to discretized value classes (e.g., current preferences). We scaled these affinity scores for each trait and each taxon between 0 (no affinity) and 1 (highest affinity; Table S5). 
Body mass and information on potential food sources were used to define the modeled food webs. The preference factors for different food-sources were derived from the fuzzy affinity scores of a taxon for a given food source (Tachet et al. 2010). We normalized the affinity scores by dividing each score by the sum of scores for all food sources for each taxon, so that the normalized scores for all food sources sum to one (Table S6). We assumed that a predator had to be larger than its potential prey by at least the value of the parameter $\pi_{\text {pred:prey }}$ (Brose et al. 2006, Woodward and Warren 2007).

Ecological preferences related to current conditions, water temperature and substrate (i.e., microhabitat) composition were used to derive factors that limit the population growth rate in the case of suboptimal environmental conditions. Similarly, if observed levels of anthropogenic stressors (pesticides and water quality related to organic matter leading to a reduction in oxygen) exceeded the tolerance of taxa, based on theSPEAR pesticides classification (Liess and von der Ohe 2005) and on the trait related to the saprobic conditions (Tachet et al. 2010; Table S5), the population death rate increased; see Performance filters for details.

Performance filters. -An implementation of trait information was already present in previous Streambugs versions (Schuwirth and Reichert 2013, Schuwirth et al. 2016) in a trait and database-specific way (i.e., for each trait and depending on the trait database used, the dependence of processes on the trait was modeled differently). To increase the generality of the model formulation, we revised the model applying the concept of performance filters (Webb et al. 2010). For a given trait $t$, these performance filters are functions that link the estimated performance currency $f_{t}$ (a dimensionless factor modifying the biological rates calculated in the model) to the normalized affinity $a_{t \text { obs }}$ that invertebrates had for the observed environmental conditions. Since we had no prior knowledge about the shape of such a relationship (except that it should be monotonic), we modeled it with a flexible exponential parametrization using two parameters for each trait $t$ : one for the intercept $\left(b_{f}\right)$ and one for the curvature $\left(c_{f_{t}}\right)$ of the performance filter (Eq. 3)

$$
f_{t}= \begin{cases}b_{f_{t}}-\left(b_{f_{t}}-1\right) \times a_{t \mathrm{obs}} & \text { if } c_{f_{t}}=0 \\ b_{f_{t}}-\left(b_{f_{t}}-1\right) \times \frac{1-\exp \left(-c_{f_{t}} \times a_{t \mathrm{obs}}\right)}{1-\exp \left(-c_{f_{t}}\right)} & \text { if } c_{f_{t}} \neq 0\end{cases}
$$

The inference covered 115 parameters, including 24 parameters common to all invertebrates and 5 that were taxon specific. The prior probability distribution $f(\boldsymbol{\theta})$ expresses prior knowledge about the parameters (Table S7). The information gained from observations is reflected in the likelihood $f(\mathbf{y} \mid \boldsymbol{\theta})$, i.e., the probability for a vector of observations $\mathbf{y}$ given the parameter values $\boldsymbol{\theta}$ (Eq. 5). The updated posterior probability distribution of parameter values $f(\boldsymbol{\theta} \mid \mathbf{y})$ expresses our belief in the parameter values $\boldsymbol{\theta}$ based on prior knowledge and observations y (Ellison 2004, Gelman et al. 2004) (Eq. 6).

Likelihood. - To allow for the comparison with observations, the deterministic results $\boldsymbol{y}^{\mathbf{m}}(\boldsymbol{\theta})\left(\mathrm{g}_{\mathrm{DM}} / \mathrm{m}\right)$ were first transformed into densities $\boldsymbol{y}^{\mathbf{m} *}(\boldsymbol{\theta})$ (individuals $/ \mathrm{m}^{2}$ ) dividing them by the river width $w$ and the average individual body mass for the invertebrate taxa or the average cell mass for algae $M$. Then, we multiplied this deterministic output with a probability to catch and correctly identify each individual $\left(p_{\text {catch }}=0.75\right)$

$$
\boldsymbol{y}^{\mathbf{m} *}(\boldsymbol{\theta})=\frac{\boldsymbol{y}^{\mathbf{m}}(\boldsymbol{\theta})}{w \times M} \times p_{\text {catch }}
$$

To consider the natural spatial aggregation of macroinvertebrates at the reach scale (patchiness), we chose a likelihood function based on the negative binomial distribution (Elliott 1971, Gray 2005), where $k$ is a dispersion parameter and $i$ represents each observation of each taxon in the community

$$
f(\boldsymbol{y} \mid \boldsymbol{\theta})=\prod_{i=1}^{n}\left[\frac{\Gamma\left(k+y_{i}\right)}{y_{i} ! \Gamma(k)} \times\left(\frac{k}{k+y_{i}^{m *}}\right)^{k} \times\left(\frac{y_{i}^{m *}}{k+y_{i}^{m *}}\right)^{y_{i}}\right]
$$

Posterior estimation. - The multivariate posterior density was numerically estimated applying a Markov Chain Monte Carlo (MCMC) approach using the Metropolis algorithm (Metropolis et al. 1953)

$$
f(\boldsymbol{\theta} \mid \boldsymbol{y}) \propto \prod_{i=1}^{n} f\left(y_{i} \mid \boldsymbol{\theta}\right) \times f(\boldsymbol{\theta})
$$

At each step $n$ of an MCMC, there is a probability that the new sample of parameter values will be accepted, only depending on the ratio of the new and the previous (i.e., $n-1)$ posterior probabilities. The jump from the $(n-1)^{\text {th }}$ to the $n^{\text {th }}$ parameter set is obtained by randomly sampling a multivariate normal distribution parameterized with a variance-covariance matrix initially defined by the prior parameter variances without correlation. To increase the efficiency of the inference and achieve the desired acceptance rate of about $23 \%$ (Gelman et al. 1996), preliminary runs were performed to optimize the jump distribution by iteratively adapting the variance component of the variance-covariance matrix.

We tested the fulfillment of the statistical assumptions of the negative binomial distribution in the likelihood function for different values of the dispersion parameter $k$ at the maximum of the posterior distribution from preliminary runs similar to Dunn and Smyth (1996; see also section S8). The final run of the inference consisted of twenty chains with 200000 simulations each, using the optimized variance-covariance matrix and a dispersion parameter $k$ of 0.7 that best satisfied the statistical assumptions of the negative binomial distribution (Fig. S8). The first half of each chain was discarded to account for burn-in effects. We assessed the convergence of the 
last half by visual inspection of trace plots, using the Heidelberger and Welch's convergence diagnostic and an estimate of the effective sample size.

\section{Model simulations}

We simulated the dynamics of the invertebrate densities in the River Sihl between April 1991 and July 1992 using the first observations as initial conditions. We propagated the prior and posterior parameter uncertainty through the model to obtain the corresponding model output uncertainty. In addition to this parameter uncertainty, we took into account observation errors (i.e., to reflect the fact that observed densities obtained by sampling patchily distributed individuals differ from the simulated deterministic densities) by sampling from the negative binomial distribution (Eq. 5) using the predicted density as mean value.

\section{Functional structure of communities}

We investigated to what degree the functional structure of simulated and observed communities match. Similar to Shipley et al. (2006), we calculated communityaggregated trait values (Eq. 7): for each category $j$ of a biological trait $t$, we multiplied the (observed or predicted) number of individuals per area $y_{i}$ of each taxon $i$ with its normalized affinity score $a_{i t_{j}}$ for this trait category $t_{j}$ (Table S6). Summarizing across taxa we then got the community-aggregated trait values $s_{t_{j}}$

$$
s_{t_{j}}=\sum_{\mathrm{i}} y_{i} \times a_{i t_{j}}
$$

To compare observed and simulated communities, we defined simulated community-aggregated trait values as underestimated or overestimated if they deviated from the trait values of observed communities by a factor of at least two (for the sake of simplicity).

All analyses were performed with the statistical software R (R Core Team 2014). We used the packages stoichcalc (Reichert and Schuwirth 2010) to derive process stoichiometries, deSolve (Soetaert et al. 2010) to solve differential equations, and coda (Plummer et al. 2006) for estimating the effective sample size and for Heidelberger and Welch's convergence diagnostic of the MCMCs.

\section{RESULTS}

\section{Community taxonomic structure}

Without calibration (i.e., using the mean of the prior), for most of the taxa, the model predictions were in the right order of magnitude, but captured few of the biomass dynamics (Figs. 2 and 3, note the prior predictions for the Ceratopogonidae, Elmidae, Empididae, and Limoniidae). For 4 out of the 13 modeled taxa, the uncalibrated model failed to predict their presence (prior predictions for Ecdyonurus, Ephemerella ignita, Leuctra, and Rhyacophila). Using Bayesian inference to calibrate the model parameters considerably improved the agreement between model predictions and observations. It also revealed that the uncertainty due to observation errors exceeded posterior parameter uncertainty (Figs. 2 and 3).

\section{Parameter inference}

The effective sample size estimated from the MCMC chains varied between 123 and 350 (Table S9). Using the stationary test of the Heidelberger \& Welch convergence criterion, all of the 115 parameters included in the inference passed the test on average for $94 \%$ of the 20 MCMC chains and, in all cases, passed the test for at least 15 chains (Table S9). These results combined with visual analysis of the chains (Fig. S10) indicated that obtained parameter distributions were a reasonable approximation of the posterior distribution.

In the prior, we assumed that the parameters were not correlated, reflecting our state of knowledge. Calculating Pearson's correlation between posterior samples, we found that the correlation for most of the 6555 parameter pairs was null or weak $\left(\left|r_{\text {all parameters }}\right|=0.06 \pm 0.06\right.$; Fig. S11).

Comparing prior and posterior distributions indicated for which parameter we could learn from the data, e.g., the bottom shear stress threshold $\tau_{\text {crit }}$ above which floodinduced drift occurs, parameters linked to the basal metabolism of invertebrates (e.g., the activation energy $E_{A}^{\text {inv }}$, the normalization constant $i_{0}^{\text {inv }}$, taxon-specific factors $f_{\text {basal tax }}$ ), and growth (taxon-specific factors $f_{\text {grotax }}$ ) (Figs. 4 and S12). In contrast, the inference did not improve our knowledge about other parameters such as the feeding efficiency $f_{\mathrm{e}}$ of the different feeding groups, and the taxon-specific food half-saturation parameters $K_{\text {food }}$ (Fig. S12) since, in our simulations, the invertebrates were not food limited (Fig. S13).

With regard to the performance filters, we generally learned less about the intercept $b_{f_{\mathrm{t}}}$ than about the curvature $c_{f_{\mathrm{t}}}$. Moreover, we did not learn about the parameters of the performance filter related to organic micropollution (Fig. S12). This was expected since the study site was considered to be unpolluted.

\section{Community functional structure}

For most of the investigated trait categories, we observed that the simulations overestimated the abundances in winter after a severe hydropeaking event (Figs. 5 and S15). The trait category "clutches in the vegetation" exhibited the largest difference between simulations and observations. However, in our taxa list, this trait category is only possessed by Ephemerella ignita. The differences between simulations and observations for this trait could be related to other traits influencing only the dynamics of E. ignita. Several trait categories 

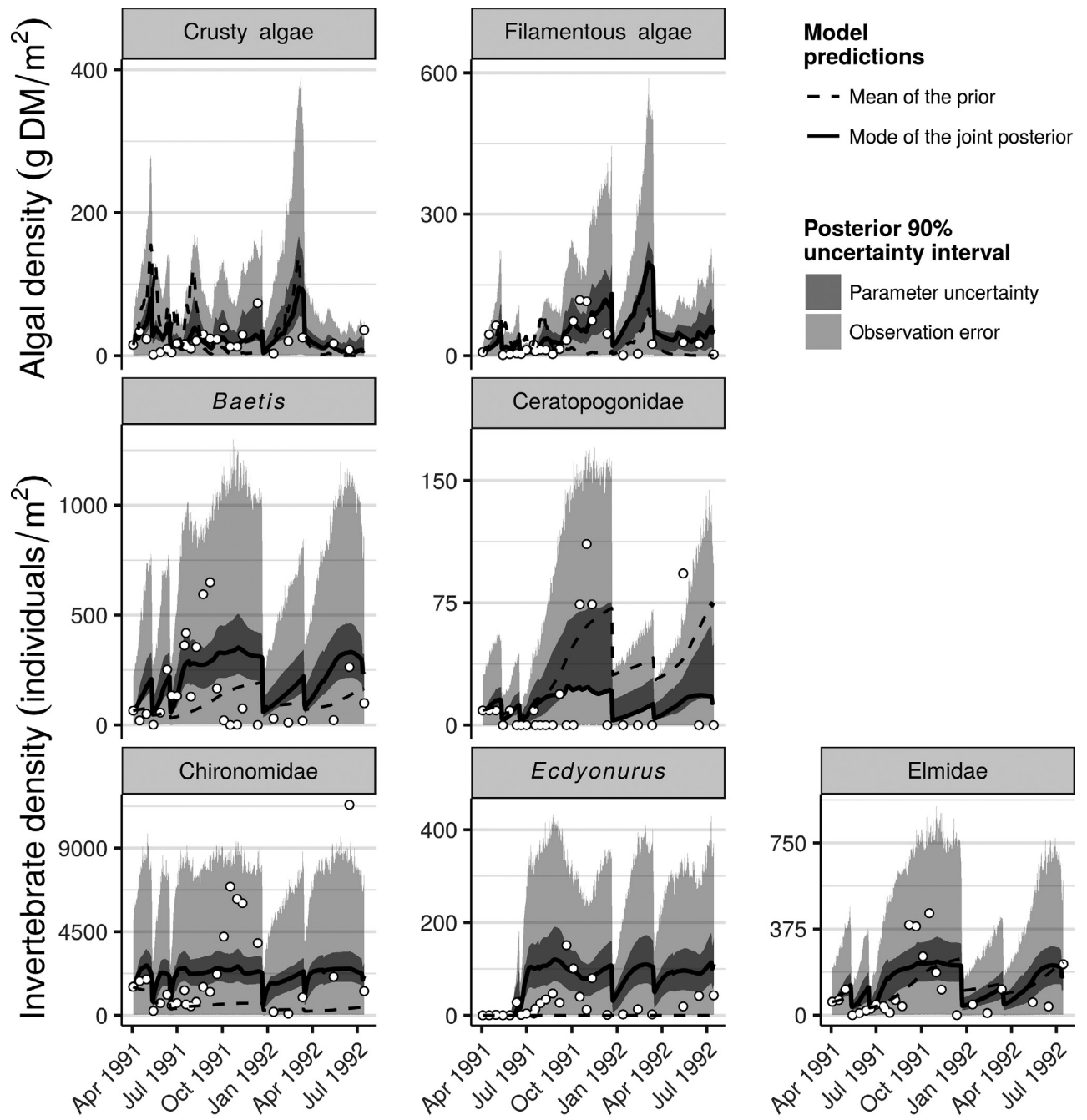

FIG. 2. Temporal evolution of observed (white dots) and simulated algae and invertebrate densities.

shared by different taxa exhibited strong differences between simulations and observations, e.g., the use of fixed isolated eggs as oviposition technique, the use of eggs as resistance forms, or a semivoltine life cycle (Fig. 5).

\section{Discussion}

Investigating how to improve predictive abilities of community ecology has been a focal topic of the past 20 years in ecology (Lawton 1999, Emmerson 2011). During this time, theoretical advances lead to the proposal of models that improved the prediction of some key aspects of communities, e.g., individual population abundances (Ott et al. 2014), food web structure (Petchey et al. 2008), or community trait profiles (Shipley et al. 2006). Here we show that the joint implementation of different general ecological theories including food webs (Pimm 1982), MTE (Brown et al. 2004), ecological stoichiometry
(Sterner and Elser 2002), and trait information in the process-based model Streambugs can help to quantitatively predict the temporal dynamics of a benthic community, which has rarely been done so far (Ings et al. 2009). The Streambugs model has recently been successfully applied to model the occurrence of macroinvertebrates in streams (Schuwirth et al. 2016) and the temporal dynamics of macroinvertebrate communities in an artificial flume experiment subjected to pesticide pulses (Kattwinkel et al. 2016). In this study we showed that this model can also be used to simulate the temporal dynamics of natural macroinvertebrate communities in streams. The processes were formulated very generally and captured a wide range of environmental influence factors to allow the model to be applied in a wide range of temperate streams. To account for dispersal limitations, the modeled taxa were chosen from the local pool of observed taxa, which were quite typical for similar rivers at the Swiss Plateau. 

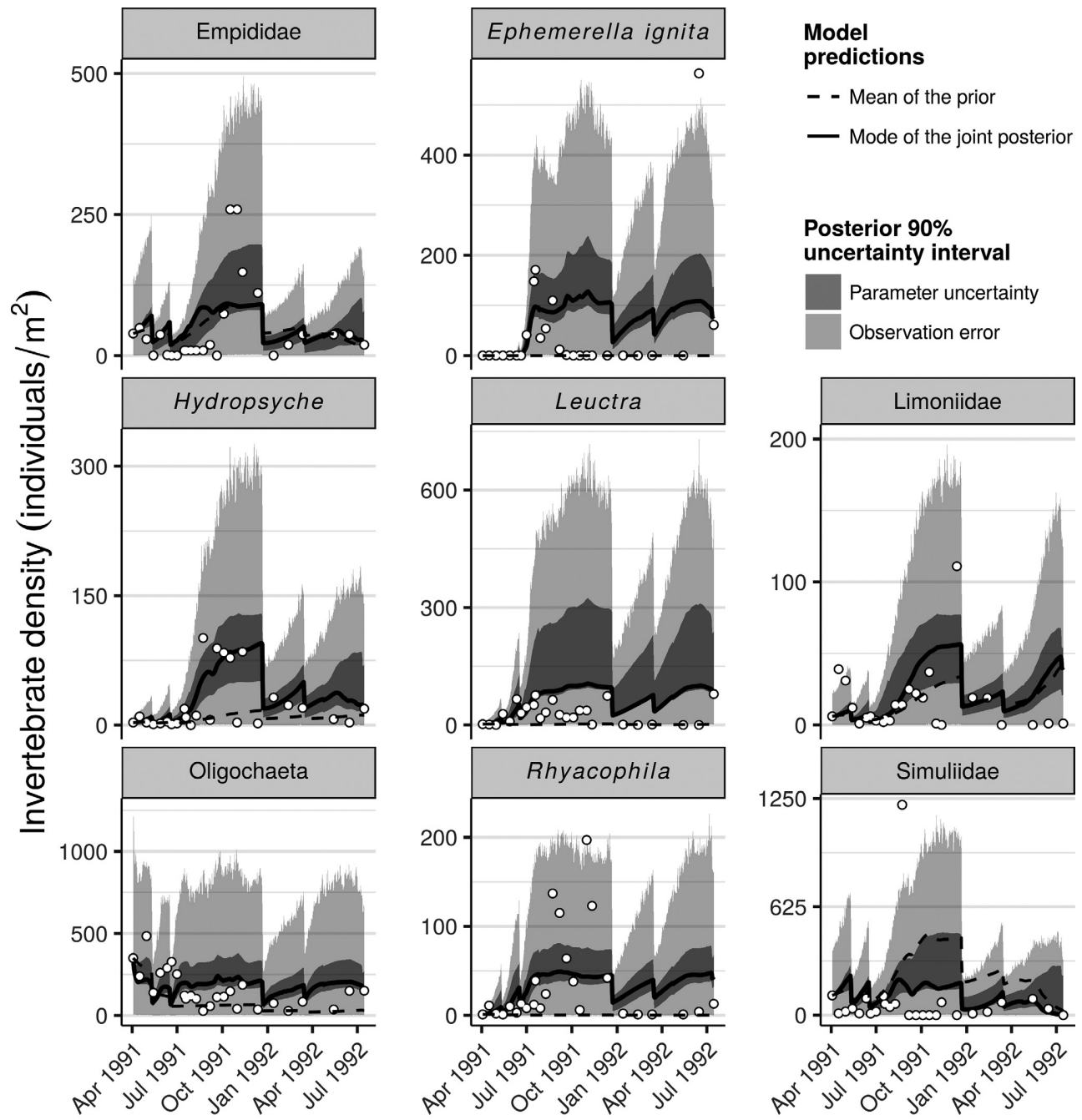

Posterior $90 \%$ uncertainty interval

Parameter uncertainty Observation error
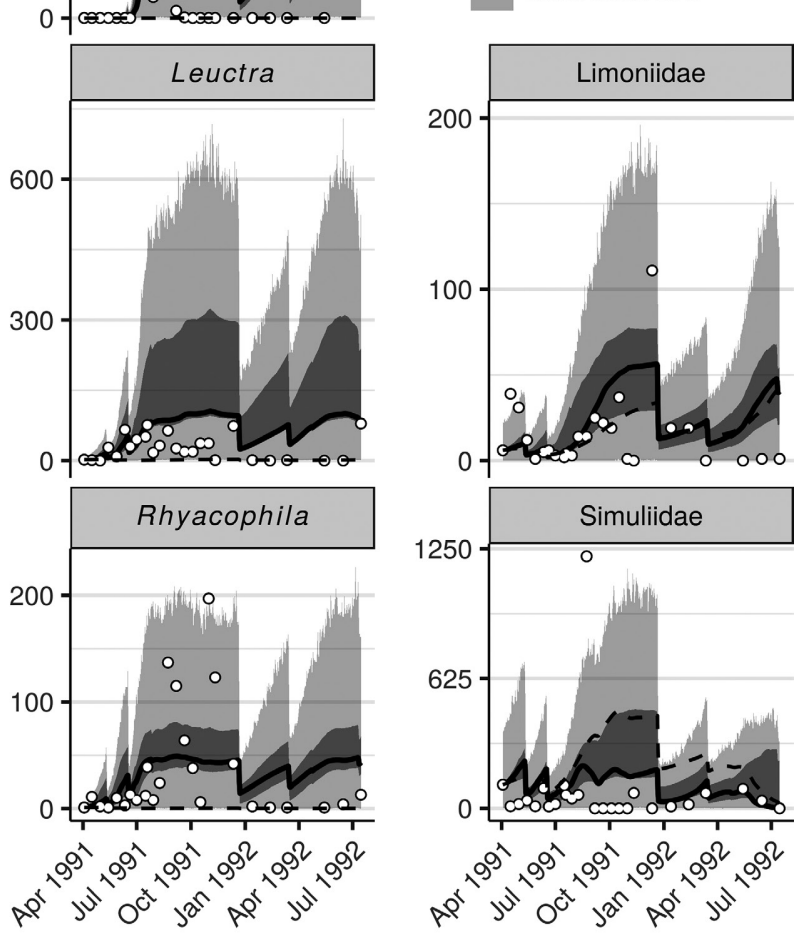

FIG. 3. Temporal evolution of observed (white dots) and simulated algae and invertebrate densities (continued).

\section{Model calibration}

As expected, Bayesian inference helped improve the model agreement with observations (Figs. 2 and 3) by updating prior knowledge about parameter values with information contained in observations (Gelman et al. 2004). Joint inference for 115 parameters was a computational challenge due to slow mixing of the Markov chains, and therefore required a high number of simulations for a reasonable coverage of the joint posterior distribution. We dealt with this by running 20 chains in parallel on separate cores (central processing units). Bayesian inference allowed gaining knowledge about several invertebrate parameters, especially those related to (1) floodinduced drift and (2) invertebrate population metabolism and growth (Fig. 4).

The drift process implemented in the Streambugs model is based on the drift implementation of the Erimo model that was applied to the same study site but using functional feeding groups as state variables (Schuwirth et al. 2008). To make it more universal, we formulated it based on bottom shear stress instead of discharge. The Bayesian inference allowed us to gain knowledge about the critical threshold $\tau_{\text {crit }}$, supporting the hypothesis of the existence of a hydraulic threshold causing bed instability responsible for massive loss of invertebrates (Gibbins et al. 2010).

According to the MTE equation (Eq. 2), three parameters were of particular importance to predict the basal metabolism of invertebrates: a normalization factor $i_{0}^{\text {inv }}$, the activation energy $E_{A}^{\text {inv }}$, and the power exponent $b^{\text {inv }}$. Regarding the parameter $b^{\text {inv }}$, the posterior distribution did not shift compared to the prior (Fig. S12), providing no support to any of the competing hypotheses about the value of this parameter: a value of $2 / 3$, reflecting surface area-to-volume (Rubner 1883), or a value of $3 / 4$, reflecting fractal organization of transport networks within organisms (Kleiber 1947, West et al. 1997). 

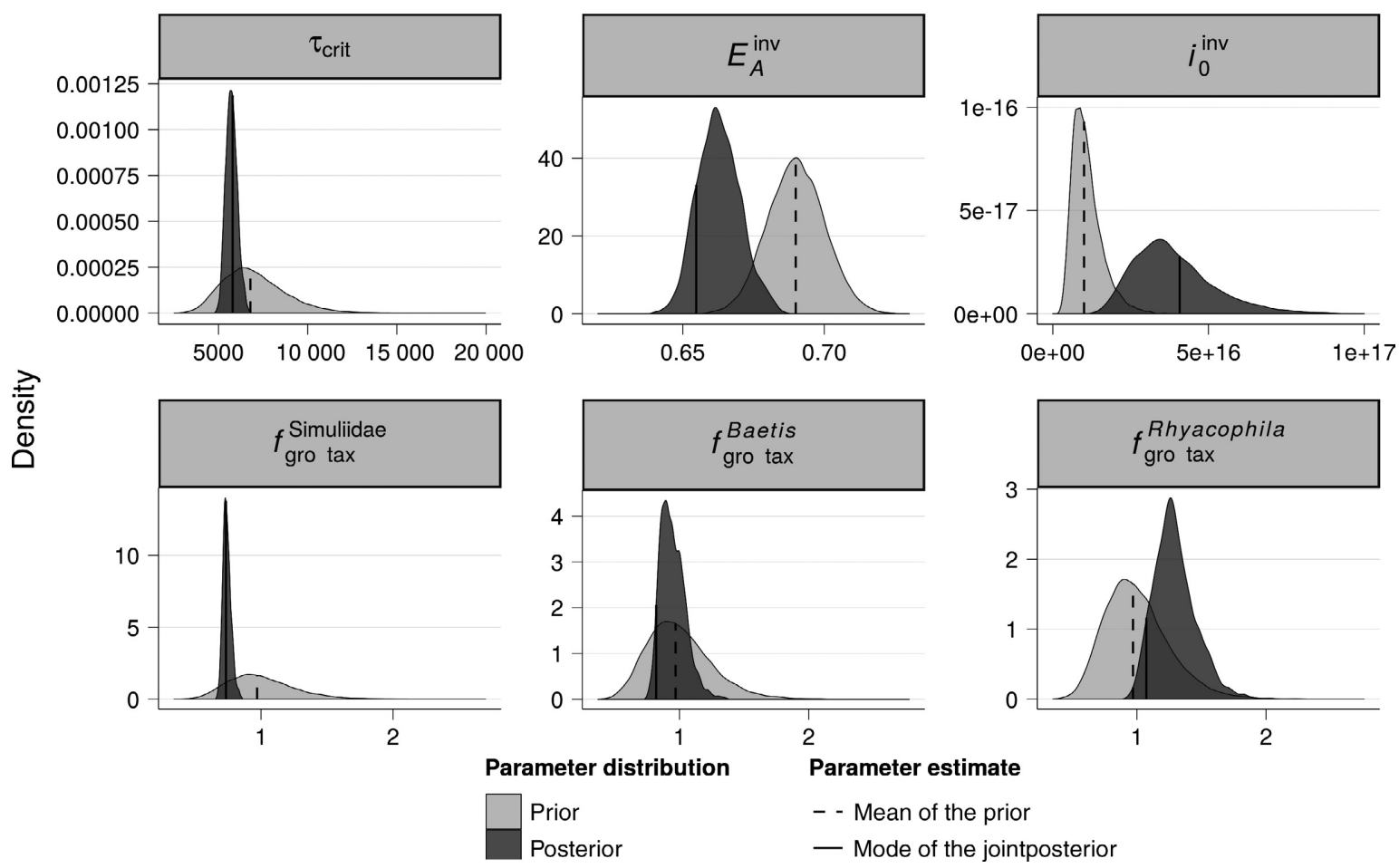

FIG. 4. Marginal prior and posterior probability distributions of six parameters: bottom shear stress threshold $\left(\tau_{\text {crit }}\right)$, metabolic activation energy $\left(E_{A}^{\text {inv }}\right)$, metabolic normalization constant $\left(i_{0}^{\text {inv }}\right)$, and growth scaling factor $\left(f_{\text {grotax }}\right)$ for three selected taxa. Units of the $x$-axes are parameter specific and provided in Table S7. Note that the parameter value at the mode of the joint posterior (straight line) does not necessarily correspond to the mode of the marginal posterior distribution.

For the two other metabolic parameters, Bayesian inference led to a shift of the posterior marginals compared to the prior distribution (Fig. 4). The marginal distribution of the activation energy $E_{A}^{\text {inv }}$ shifted to lower values, indicating a faster increase of the metabolic rate with increasing temperatures (Fig. S15a). The posterior distribution obtained (best parameter value: 0.65 ) is different from the prior estimates defined for all invertebrates according to Ehnes et al. (2011) but (1) still within the range proposed for organisms with aerobic respiration (0.6-0.7; Gillooly et al. 2002) and (2) similar to the $E_{\mathrm{A}}$ values corresponding to insects obtained by Ehnes et al. (2011) using direct measurements $(0.66 \pm 0.04)$. The posterior distribution of the normalization factor $i_{0}^{\text {inv }}$ was shifted toward larger values compared to the prior, but after converting to the same units used in Ehnes et al. (2011) (from $\mathrm{J} / \mathrm{yr}$ to $\log$-transformed $\mathrm{J} / \mathrm{s}$ ), we found that our best estimate (20.98) was in the range of the values they obtained for insects $(21.97 \pm 1.58)$. This difference between $i_{0}$ estimates for all invertebrates and insects alone suggests phylogenetic or taxonomic differences (Ehnes et al. 2011). Samples from the posterior distribution of $E_{A}^{\text {inv }}$ and $i_{0}^{\text {inv }}$ were positively correlated (Pearson's $r=0.74$ Table S11). Based on the MTE's equation (Eq. 2), this suggests that, for large values of $E_{A}^{\text {inv }}$, which decrease the basal metabolic rate (Fig. S15a), large values of $i_{0}^{\text {inv }}$ are required to compensate for this decrease (Fig. S15b).
The mean posterior correlations among the $f_{\text {gro tax }}$ parameters of the different invertebrates $\left.\left|r_{f_{\text {gro tax }}^{\text {in }}}\right|=0.21 \pm 0.11\right)$ were above the average of all parameters $\left(\left|r_{\text {all parameters }}\right|=\right.$ $0.06 \pm 0.06$; Fig. S11). This indicates that the biotic interactions between the populations of different taxa play a role in the dynamics of benthic communities (Schuwirth et al. 2016). Moreover, the marginal posterior distributions of these parameters differ between taxa (Figs. 4 and S12), being either (1) not different from the prior (e.g., for Baetis) or shifted toward (2) smaller values (e.g., for Simuliidae) or (3) larger values (e.g., for Rhyacophila). These results indicate that differences among taxa exist even if the growth rate is scaled on the basal metabolism. These differences could be related to, for example, their elemental composition (Sterner and Elser 2002).

Trait-based approaches have recently received increasing attention due to their potential ability to improve the predictions in community ecology (McGill et al. 2006). This potential is based on the hypothesis that the environment acts as a filter on organisms through their traits (Southwood 1977), a hypothesis that recently received increasing support (Jung et al. 2010, Mondy and Usseglio-Polatera 2014), e.g., also in the context of multiple stressors (Mondy et al. 2016). This has led to the development of promising approaches to predict key features of communities (Shipley et al. 2006, Laughlin et al. 2012). A straightforward way of using 

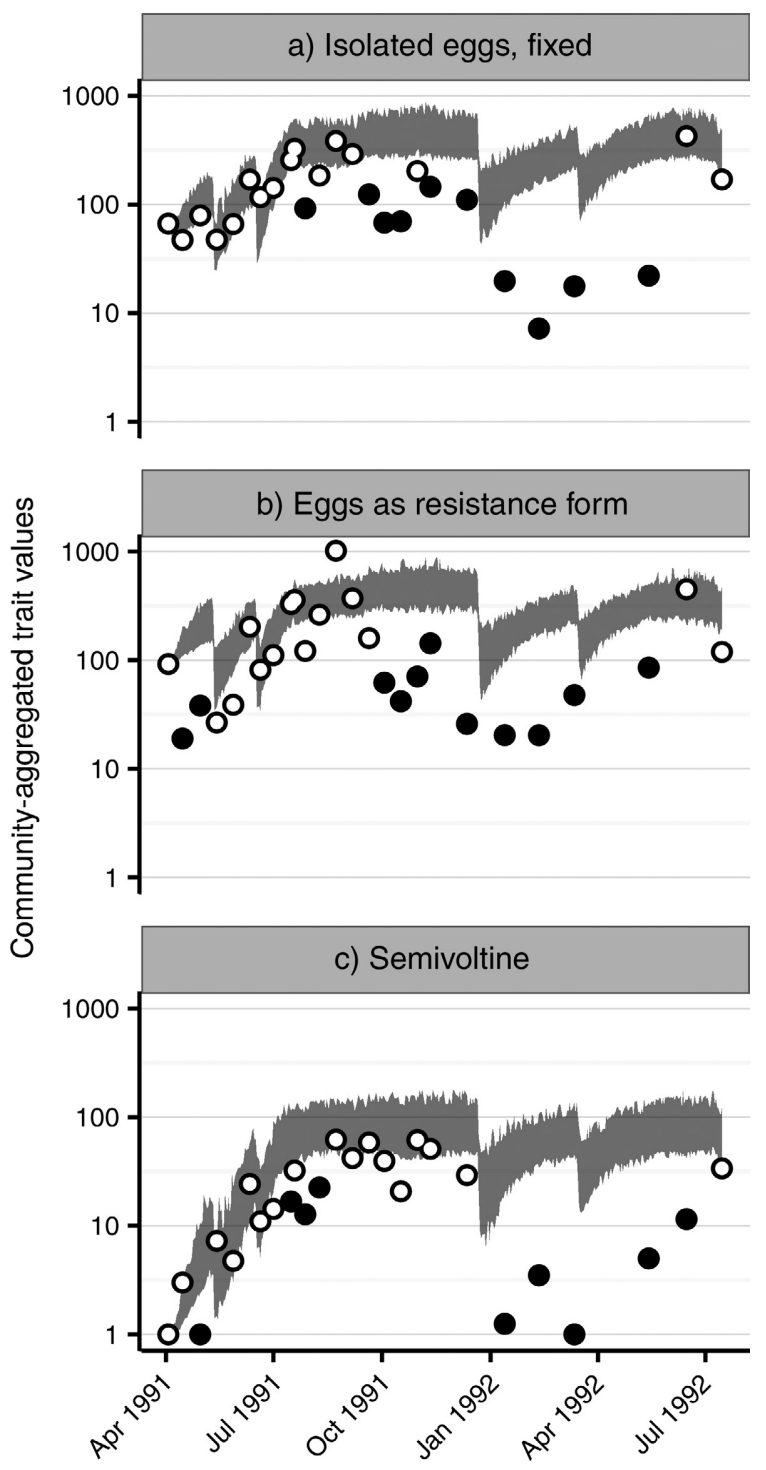

FIG. 5. Temporal evolution of community-aggregated trait values for simulated (gray area) and observed (white and black dots) communities for the trait categories "fixed, isolated eggs," "eggs as resistance form," and "semivoltinism". Black dots correspond to values from observed communities that deviate by at least a factor of two from values of simulated communities.

traits in a process-based model such as Streambugs is to use functions relating organisms' fitness (performance currency) to their traits in a given environment (Webb et al. 2010). The inference of these performance filter parameters (Fig. S12) improved our knowledge mostly about the curvature and less about the intercept (i.e., the performance corresponding to no affinity for observed environmental conditions; Fig. 6). According to our study, larger affinity scores are required to reach good fitness levels ( $f_{\mathrm{t}}$ factors close to 1$)$ for maximum temperature and current (negative $c_{f}$ ) than for substrates (positive $c_{f} ;$ Fig. 6). This seems to indicate that the effects of maximum temperature and current

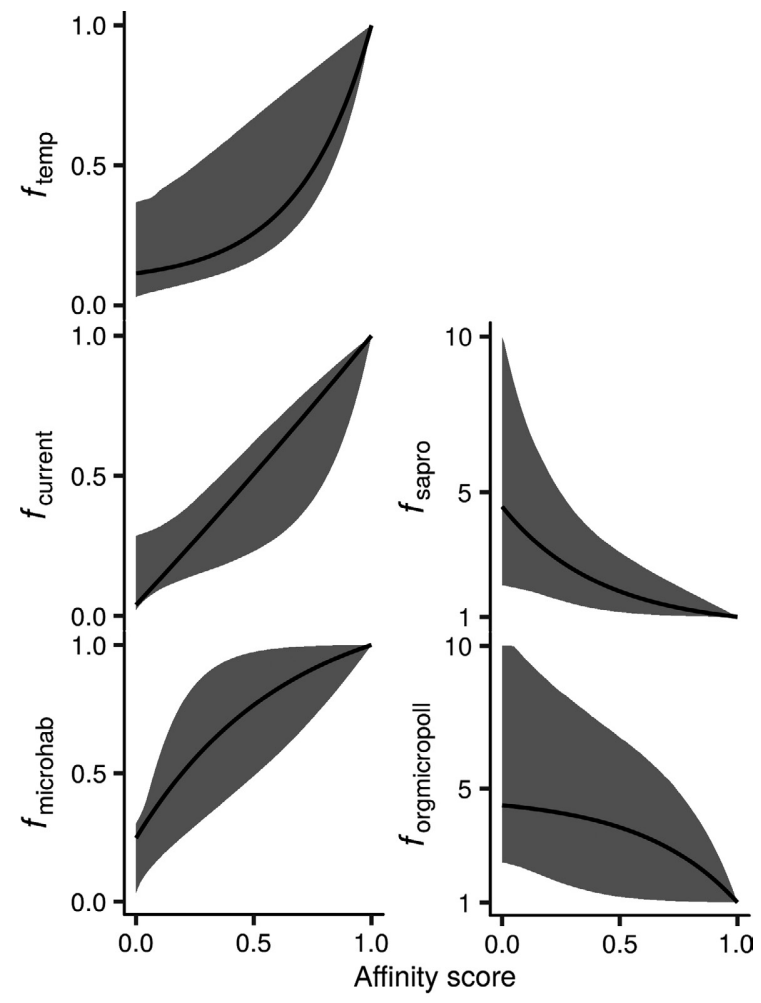

FIG. 6. Shape and uncertainty (gray area) of the performance filters with posterior values (mode of the joint posterior; black line) of the intercept and curvature parameters for maximum temperature $\left(f_{\text {temp }}\right)$, current $\left(f_{\text {current }}\right)$, substrate $\left(f_{\text {microhab }}\right)$, saprobic conditions $\left(f_{\text {sapro }}\right)$, and presence of organic micropollutants ( $f_{\text {orgmicropoll }}$ ).

conditions on benthic communities are stronger than the effect of substrate composition (Fig. S13). This is in agreement with some previous studies (Statzner et al. 1988, Mérigoux and Dolédec 2004) whereas other studies attribute a more important role to the substrate composition (Quinn and Hickey 1990, Beisel et al. 1998). It is thus not clear if a general pattern explaining the relative effects of current and substrate on benthic communities exists. Most likely this depends on the study area.

The majority of organism data used in this study was not available at the species level. Therefore, we had to use identification levels coarser than species in the model. This has probably lowered the model efficiency since the ecological characteristics may vary significantly among different species of a higher taxonomic unit.

In this study, we dealt with this problem by averaging the trait information of the different members (e.g., species) of the same taxon (e.g., genus). In the specific case of the SPEAR pesticides $_{\text {trait, we considered a taxon as }}$ insensitive if at least one of its members was classified as insensitive. The propagation of the posterior performance filter parameter uncertainty to the model outputs allows us to estimate a response space likely to include the response of species actually living in the site. More precise 
taxonomic information would help in reducing uncertainty about the performance filter parameters and reducing the model output uncertainty. For practical reasons, biomonitoring data of macroinvertebrates is often not resolved to species level for all taxa. The development of DNA barcoding might help resolve this issue in the future (Carew et al. 2013).

\section{Analysis of model deficiencies}

By definition, all environmental models are wrong (Box and Draper 1987) since they are always a simplification of complex natural systems. Therefore, even if the Streambugs results are promising, we need to analyze how the predictions deviate from observations and try to understand the origin of these differences to improve the model and our understanding of the underlying processes. The analysis of community-aggregated biological trait values helped to analyze potential reasons for model deficiencies.

Macroinvertebrate reproduction strategies seem to be related, at least to some extent, to these differences. Indeed, taxa that are using fixed isolated eggs (e.g., Ephemerella ignita, Leuctra, and Rhyacophila) as part of a life history strategy are often overestimated in the model simulations compared to observations (Figs. 3 and 5). Repeated hydropeaking events during spring and summer, as observed in our case study (Fig. 1), can impair the reproductive success of amphibiotic insects. These events can completely modify the habitat structure of river reaches by displacing or submerging rocks that are preferentially selected by some invertebrates for oviposition (Encalada and Peckarsky 2006).

Moreover, in the winter season, invertebrates have a lower metabolism due to low water temperatures (Brown et al. 2004) and they can use resistance forms to withstand these harsher environmental conditions. For example, the mayfly Ephemerella ignita is known to have a highly synchronized, univoltine life history with a long overwintering egg diapause period and a short, fast-growing, summer larval development (Maitland 1965, Lancaster et al. 2011). Without differentiating life stages, the model is not able to describe these seasonal occurrence patterns. The implementation of different life stages is challenging (Eckman 1996) but might be helpful for a more realistic description of community dynamics, especially regarding insects.

In our study, the frequency of hydropeaking events was lower in winter compared to spring and summer. However, the largest event occurred on 22 December 1991 (Fig. 1) and the Streambugs simulations failed to reflect the strong decrease and slow recovery of abundances observed for most of the taxa and trait categories (Figs. 2, 3, 5, and S14). This is especially the case for taxa considered as semi/univoltine (e.g., Ecdyonurus, Leuctra, and Rhyacophila; Tachet et al. 2010) that have a lower number of generations per year compared to taxa considered as plurivoltine (e.g., Baetis, most of the Chironomidae and Simuliidae; Tachet et al. 2010). The ability to produce several generations per year is an important mechanism to increase tolerance against many different anthropogenic stressors (e.g., Liess and von der Ohe 2005, Mondy et al. 2016).

Finally, we decided to omit taxa with low occurrences and abundances because the chosen model approach does not account for demographic stochasticity. Demographic stochasticity becomes more important, the smaller the population becomes (Lande et al. 2003), while it becomes negligible for large populations due to the law of large numbers. Extending our model to a stochastic model would be desirable, especially regarding the inclusion of rare taxa. However, this would increase model complexity and the computational burden considerably. This makes Bayesian inference very challenging (but see Kattwinkel and Reichert 2017, for recent progress on this topic).

\section{Effects of hydrological alterations}

Water abstraction can lead to increased algal biomass and fine sediment deposition in residual flow reaches due to a decrease in flow velocity and a reduction of bedmoving floods. Both effects can impact the macroinvertebrate community in addition to direct effects of an altered flow regime. While effects of water abstraction on macroinvertebrate abundance varies between taxa (leading to a species turnover), invertebrate richness commonly decreases due to a decrease in habitat diversity (Dewson et al. 2007). Artificial floods are conducted to mitigate these effects in regulated rivers downstream of dams and can serve as large-scale experiments (Konrad et al. 2011, Olden et al. 2014). While increased drift and reduced densities are commonly observed (short-term) responses of macroinvertebrates to experimental floods (Gillespie et al. 2015), studies on long-term effects (Robinson 2012) and on the temporal dynamics of effects are rare. In the Sihl, the artificial floods exceeding the critical bottom shear stress for bed movement decreased the algal biomass and fine sediment coverage, especially when occurring in spring and summer (Figs. 1 and 2). In the model, biomass dynamics of the macroinvertebrates were most directly influenced by drift induced by bedmoving floods. But also indirect effects of algae and fine sediment coverage that influence the habitat suitability via substrate availability are visible (Fig. S13). The analysis of community-aggregated trait profiles suggests that recovery dynamics of macroinvertebrates depend on their reproductive live history strategy (Fig. 5) in concordance with Kennedy et al. (2016) who found a strong influence of adult egg-laying behavior on the resistance of aquatic insects to hydropeaking. Therefore, the timing of artificial flood events is crucial and should mimic the natural dynamics of the river to support a re-establishment of a near natural community structure.

\section{Conclusions and perspectives}

We showed that integrating several ecological theories (MTE, ecological stoichiometry, food web theory) and 
using trait-based performance filters in the same framework allows a further step to be taken toward a better understanding and prediction of the dynamics of natural macroinvertebrate communities in streams, which is an extremely challenging task. We think that this general approach is transferable to a wide range of ecosystems and other taxonomic systems, while the process formulations would need to be adapted to the modeled system. However, process-based models such as Streambugs rely on many uncertain parameters for which prior information is scarce. It is thus of the utmost importance to use such models within a framework that allows this uncertainty to be considered and propagated, such as Bayesian inference (Ellison 2004).

Analyses of community-aggregated trait values helped generate ideas for further improvement of the process formulations to increase the predictive capabilities of the model. In particular, accounting for different life stages through the integration of biological life-history traits seems to be of the highest priority in order to better describe the population dynamics of aquatic insects and their response to disturbances such as hydropeaking. This might also contribute to unravelling the reasons for differences in posterior estimates for specific growth rates that go beyond metabolic scaling. To include rare taxa, the model approach would need to be extended to account for stochasticity. Finally, as observation error turned out to be a major contribution to total model output uncertainty, we would strongly recommend increasing the sampling area as well as the taxonomic resolution of the biological data for model calibration so as to reduce the effect of patchiness and to decrease parameter uncertainty. A thorough description and reduction of model output uncertainty is a necessary step if we want to use such models to further improve our mechanistic understanding and to support environmental management.

\section{ACKNOWLEDGMENTS}

We thank Peter Reichert for developing the test of the fulfillment of the assumptions of the negative binomial distribution as well as for stimulating discussions. We thank Mira Kattwinkel and Carlo Albert for their comments on previous versions of the manuscript. We thank Andrew Clarke for language revision. We also thank Astrid Schmidt-Kloiber and an anonymous reviewer for their constructive and valuable comments that contributed to improve this manuscript.

\section{Literature Cited}

Allen, A. P., and J. F. Gillooly. 2009. Towards an integration of ecological stoichiometry and the metabolic theory of ecology to better understand nutrient cycling. Ecology Letters 12:369-384.

Ascough, J. C., H. R. Maier, J. K. Ravalico, and M. W. Strudley. 2008. Future research challenges for incorporation of uncertainty in environmental and ecological decisionmaking. Ecological Modelling 219:383-399.

Beisel, J.-N., P. Usseglio-Polatera, S. Thomas, and J.-C. Moreteau. 1998. Stream community structure in relation to spatial variation: the influence of mesohabitat characteristics. Hydrobiologia 389:73-88.
Boit, A., N. D. Martinez, R. J. Williams, and U. Gaedke. 2012. Mechanistic theory and modelling of complex food-web dynamics in Lake Constance. Ecology Letters 15:594-602.

Box, G. E. P., and N. R. Draper. 1987. Empirical model-building and response surfaces. John Wiley \& Sons, Oxford, UK.

Brose, U., R. J. Williams, and N. D. Martinez. 2006. Allometric scaling enhances stability in complex food webs. Ecology Letters 9:1228-1236.

Brown, J. H., J. F. Gillooly, A. P. Allen, V. M. Savage, and G. B. West. 2004. Toward a metabolic theory of ecology. Ecology 85:1771-1789.

Brown, L. E., F. K. Edwards, A. M. Milner, G. Woodward, and M. E. Ledger. 2011. Food web complexity and allometric scaling relationships in stream mesocosms: Implications for experimentation. Journal of Animal Ecology 80:884-895.

Carew, M. E., V. J. Pettigrove, L. Metzeling, and A. A. Hoffmann. 2013. Environmental monitoring using next generation sequencing: rapid identification of macroinvertebrate bioindicator species. Frontiers in Zoology 10:1-15.

Chevenet, F., S. Dolédec, and D. Chessel. 1994. A fuzzy coding approach for the analysis of long-term ecological data. Freshwater Biology 31:295-309.

Dewson, Z. S., A. B. W. James, and R. G. Death. 2007. A review of the consequences of decreased flow for instream habitat and macroinvertebrates. Journal of the North American Benthological Society 26:401-415.

Dunn, P. K., and G. K. Smyth. 1996. Randomized quantile residuals. Journal of Computational and Graphical Statistics 5:236-244.

Eckman, J. E. 1996. Closing the larval loop: linking larval ecology to the population dynamics of marine benthic invertebrates. Journal of Experimental Marine Biology and Ecology 200:207-237.

Ehnes, R. B., B. C. Rall, and U. Brose. 2011. Phylogenetic grouping, curvature and metabolic scaling in terrestrial invertebrates. Ecology Letters 14:993-1000.

Elliott, J. M. 1971. Some methods for the statistical analysis of samples of benthic invertebrates. Freshwater Biological Association, Ambleside, UK.

Ellison, A. M. 2004. Bayesian inference in ecology. Ecology Letters 7:509-520.

Emmerson, M. C. 2011. The predictive science of community ecology. Journal of Animal Ecology 80:1111-1114.

Encalada, A. C., and B. L. Peckarsky. 2006. Selective oviposition of the mayfly Baetis bicaudatus. Oecologia 148: 526-537.

Gelman, A., G. Roberts, and W. Gilks. 1996. Efficient metropolis jumping hules. Bayesian Statistics 5:42.

Gelman, A., J. B. Carlin, H. S. Stern, and D. B. Rubin. 2004. Bayesian data analysis. Texts in statistical science series. Chapman \& Hall/CRC, Boca Raton, Florida, USA.

Gibbins, C., D. Vericat, and R. J. Batalla. 2007. When is stream invertebrate drift catastrophic? The role of hydraulics and sediment transport in initiating drift during flood events. Freshwater Biology 52:2369-2384.

Gibbins, C., R. J. Batalla, and D. Vericat. 2010. Invertebrate drift and benthic exhaustion during disturbance: Response of mayflies (Ephemeroptera) to increasing shear stress and riverbed instability. River Research and Applications 26: 499-511.

Gillespie, B. R., S. Desmet, P. Kay, M. R. Tillotson, and L. E. Brown. 2015. A critical analysis of regulated river ecosystem responses to managed environmental flows from reservoirs. Freshwater Biology 60:410-425.

Gillooly, J. F., E. L. Charnov, G. B. West, V. M. Savage, and J. H. Brown. 2002. Effects of size and temperature on developmental time. Nature 417:70-73. 
Gray, B. R. 2005. Selecting a distributional assumption for modelling relative densities of benthic macroinvertebrates. Ecological Modelling 185:1-12.

Ings, T. C., et al. 2009. Review: ecological networks-beyond food webs. Journal of Animal Ecology 78:253-269.

Jung, V., C. Violle, C. P. Mondy, L. Hoffmann, and S. Muller. 2010. Intraspecific variability and trait-based community assembly. Journal of Ecology 98:1134-1140.

Kattwinkel, M., and P. Reichert. 2017. Bayesian parameter inference for individual-based models using a Particle Markov Chain Monte Carlo method. Environmental Modelling and Software 87:110-119.

Kattwinkel, M., P. Reichert, J. Rüegg, M. Liess, and N. Schuwirth. 2016. Modeling macroinvertebrate community dynamics in stream mesocosms contaminated with a pesticide. Environmental Science and Technology 50: 3165-3173.

Kendall, B. E., C. J. Briggs, W. W. Murdoch, P. Turchin, S. P. Ellner, E. McCauley, R. M. Nisbet, and S. N. Wood. 1999. Why do populations cycle? A synthesis of statistical and mechanistic modeling approaches. Ecology 80:1789-1805.

Kennedy, T. A., J. D. Muehlbauer, C. B. Yackulic, D. A. Lytle, S. W. Miller, K. L. Dibble, E. W. Kortenhoeven, A. N. Metcalfe, and C. V. Baxter. 2016. Flow management for hydropower extirpates aquatic insects, undermining river food webs. BioScience 66:561-575.

Kleiber, M. 1947. Body size and metabolic rate. Physiological Reviews 27:511-541.

Konrad, C. P., et al. 2011. Large-scale flow experiments for managing river systems. BioScience 61:948-959.

Lancaster, J., B. J. Downes, and A. Arnold. 2011. Lasting effects of maternal behaviour on the distribution of a dispersive stream insect. Journal of Animal Ecology 80:1061-1069.

Lande, R., S. Engen, and B.-E. Sæther. 2003. Stochastic population dynamics in ecology and conservation. Oxford University Press, Oxford, UK.

Laughlin, D. C., C. Joshi, P. M. Bodegom, Z. A. Bastow, and P. Z. Fulé. 2012. A predictive model of community assembly that incorporates intraspecific trait variation. Ecology Letters 15:1291-1299.

Lawton, J. H. 1999. Are there general laws in ecology? Oikos 84:177-192.

Liess, M., and P. C. von der Ohe. 2005. Analyzing effects of pesticides on invertebrate communities in streams. Environmental Toxicology and Chemistry 24:954-965.

MacArthur, R. H. 1984. Geographical ecology: Patterns in the distribution of species. Princeton University Press, Princeton, New Jersey, USA.

Maitland, P. S. 1965. The distribution, life cycle, and predators of Ephemerella ignita (Poda) in the River Endrick, Scotland. Oikos 16:48-57.

McGill, B. J., B. J. Enquist, E. Weiher, and M. Westoby. 2006. Rebuilding community ecology from functional traits. Trends in Ecology and Evolution 21:178-185.

Mérigoux, S., and S. Dolédec. 2004. Hydraulic requirements of stream communities: a case study on invertebrates. Freshwater Biology 49:600-613.

Metropolis, N., A. W. Rosenbluth, M. N. Rosenbluth, A. H. Teller, and E. Teller. 1953. Equation of state calculations by fast computing machines. Journal of Chemical Physics 21:1087-1092.

Moe, S. J., R. S. Stelzer, M. R. Forman, W. S. Harpole, T. Daufresne, and T. Yoshida. 2005. Recent advances in ecological stoichiometry: insights for population and community ecology. Oikos 109:29-39.

Mondy, C. P., and P. Usseglio-Polatera. 2014. Using fuzzy-coded traits to elucidate the non-random role of anthropogenic stress in the functional homogenisation of invertebrate assemblages. Freshwater Biology 59:584-600.

Mondy, C. P., I. Muñoz, and S. Dolédec. 2016. Life-history strategies constrain invertebrate community tolerance to multiple stressors: a case study in the Ebro basin. Science of the Total Environment 572:196-206.

Olden, J. D., et al. 2014. Are large-scale flow experiments informing the science and management of freshwater ecosystems? Frontiers in Ecology and the Environment 12: 176-185.

Ott, D., C. Digel, B. C. Rall, M. Maraun, S. Scheu, and U. Brose. 2014. Unifying elemental stoichiometry and metabolic theory in predicting species abundances. Ecology Letters 17:1247-1256.

Petchey, O. L., A. P. Beckerman, J. O. Riede, and P. H. Warren. 2008. Size, foraging, and food web structure. Proceedings of the National Academy of Sciences USA 105:4191-4196.

Pimm, S. L. 1982. Food webs. Springer, Netherlands.

Plummer, M., N. Best, K. Cowles, and K. Vines. 2006. CODA: convergence diagnosis and output analysis for MCMC. R News 6:7-11.

Price, C. A., J. F. Gilooly, A. P. Allen, J. S. Weitz, and K. J. Niklas. 2010. The metabolic theory of ecology: prospects and challenges for plant biology. New Phytologist 188:696-710.

Quinn, J. M., and C. W. Hickey. 1990. Magnitude of effects of substrate particle size, recent flooding, and catchment development on benthic invertebrates in 88 New Zealand Rivers. New Zealand Journal of Marine and Freshwater Research 24:411-427.

R Core Team. 2014. R: A language and environment for statistical computing. R Foundation for Statistical Computing, Vienna, Austria. https://www.R-project.org

Reichert, P., and N. Schuwirth. 2010. A generic framework for deriving process stoichiometry in environmental models. Environmental Modelling and Software 25:1241-1251.

Robinson, C. T. 2012. Long-term changes in community assembly, resistance, and resilience following experimental floods. Ecological Applications 22:1949-1961.

Rubner, M. 1883. Über den Einfluss der Körpergrösse auf Stoff- und Kraftwechsel. Zeitschrift für Biologie 19:535-562.

Scheiner, S. M., and M. R. Willig. 2008. A general theory of ecology. Theoretical Ecology 1:21-28.

Schoener, T. W. 1986. Mechanistic approaches to community ecology: A new reductionism. American Zoologist 26: 81-106.

Schuwirth, N., and P. Reichert. 2013. Bridging the gap between theoretical ecology and real ecosystems: modeling invertebrate community composition in streams. Ecology 94: 368-379.

Schuwirth, N., M. Kühni, S. Schweizer, U. Uehlinger, and P. Reichert. 2008. A mechanistic model of benthos community dynamics in the River Sihl, Switzerland. Freshwater Biology 53:1372-1392.

Schuwirth, N., A. Dietzel, and P. Reichert. 2016. The importance of biotic interactions for the prediction of macroinvertebrate communities under multiple stressors. Functional Ecology 30:974-984.

Shipley, B., D. Vile, and É. Garnier. 2006. From plant traits to plant communities: a statistical mechanistic approach to biodiversity. Science 314:812-814.

Soetaert, K., T. Petzoldt, and R. W. Setzer. 2010. Solving differential equations in R: Package deSolve. Journal of Statistical Software 33:1-25.

Southwood, T. R. E. 1977. Habitat, the templet for ecological strategies? Journal of Animal Ecology 46:337-365.

Statzner, B., J. A. Gore, and V. H. Resh. 1988. Hydraulic stream ecology: Observed patterns and potential applications. 
Journal of the North American Benthological Society 7: 307-360.

Sterner, R. W., and J. J. Elser. 2002. Ecological stoichiometry: the biology of elements from molecules to the biosphere. Princeton University Press, Princeton, New Jersey, USA.

Tachet, H., P. Richoux, M. Bournaud, and P. Usseglio-Polatera. 2010. Invertébrés d'eau douce: Systématique, biologie, écologie. Third edition. CNRS éditions, Paris, France.

Webb, C. T., J. A. Hoeting, G. M. Ames, M. I. Pyne, and N. LeRoy Poff. 2010. A structured and dynamic framework to advance traits-based theory and prediction in ecology. Ecology Letters 13:267-283.

West, G. B., J. H. Brown, and B. J. Enquist. 1997. A general model for the origin of allometric scaling laws in biology. Science 276:122-126.

Woodward, G., and P. H. Warren. 2007. Body size and predatory interactions in freshwaters: scaling from individuals to communities. Pages $98-117$ in A. G. Hildrew, D. Raffaelli and R. Edmonds-Brown, editors. In Body Size: The Structure and Function of Aquatic Ecosystems. Cambridge University Press, Cambridge, UK

\section{SUPPORTING INFORMATION}

Additional supporting information may be found in the online version of this article at http://onlinelibrary.wiley.com/ doi/10.1002/eap.1530/full 